\title{
Enhanced Simultaneous Distributed Strain and Temperature Fiber Sensor Employing Spontaneous Brillouin Scattering and Optical Pulse Coding
}

\author{
Marcelo A. Soto, Gabriele Bolognini, Member, IEEE, and Fabrizio Di Pasquale, Member, IEEE
}

\begin{abstract}
In this work, we propose the use of optical pulse coding techniques for simultaneous strain and temperature sensing based on spontaneous Brillouin scattering. Optical pulse coding provides a significant receiver signal-to-noise ratio enhancement, allowing for accurate Brillouin intensity and frequency shift measurements at low peak power levels. Due to the cross-sensitivity of these two parameters on both temperature and strain, optical pulse coding improves the temperature and strain resolution and sensing range with respect to Brillouin sensors operating with conventional single-pulses at the same peak power level. The proposed technique provides a high-performance cost-effective solution avoiding the use of high peak power levels.
\end{abstract}

Index Terms-Brillouin scattering, modulation coding, optical fiber measurement, optical time-domain reflectometry (OTDR).

\section{INTRODUCTION}

A PPLICATIONS of optical fiber sensors have been growing rapidly in the last years [1]. In particular, distributed fiber-optic sensors (DFS) are attracting a lot of attention for industrial applications, environmental and structural-health monitoring, offering a unique and truly distributed sensing mechanism for long-range applications.

Raman or Brillouin scattering are the most widely exploited effects in distributed optical sensing, and are often used together with optical time-domain reflectometry (OTDR) [2], [3]. Compared to Raman scattering effect, which is dependent on temperature only, Brillouin scattering allows in principle for simultaneous temperature and strain measurements within the same optical fiber [4]. Brillouin-based DFS can be implemented using either spontaneous Brillouin scattering (SpBS) or stimulated Brillouin scattering (SBS) effects and generally require measurements of both intensity and frequency shift. Although SBSbased measurements require access to both fiber ends and more complex receiver schemes [2], they generally allow for more accurate temperature and strain resolution. On the other hand, SpBS-based DFS allow for simpler implementations, require access to one fiber end only, and are not affected by nonlocal effects [5], thus appearing more suitable for long-range sensing applications.

Note that when SpBS is used for simultaneous strain and temperature sensing within the same optical fiber, the achievable accuracy is mainly limited by the noise affecting the intensity measurement [4]. In this letter, we experimentally show

\footnotetext{
Manuscript received September 30, 2008; revised November 06, 2008. First published February 03, 2009; current version published March 18, 2009.

The authors are with Scuola Superiore Sant'Anna, Pisa 56124, Italy (e-mail: m.soto@sssup.it; gabriele.bolognini@sssup.it; fabrizio.dipasquale@sssup.it).

Digital Object Identifier 10.1109/LPT.2009.2012874
}

that pulse coding, applied to SpBS-based DFS for combined measurements of intensity and frequency shift, allows for accurate simultaneous strain and temperature sensing, providing a high-performance and cost-effective solution.

\section{THEORY}

In SpBS-based DFS, the Brillouin spectrum center-frequency shift (BFS) and Brillouin intensity parameters depend on both strain and temperature. This feature allows for sensing both physical variables simultaneously by measuring, through OTDR-based techniques, both BFS and Brillouin intensity traces. The Landau-Placzek ratio [3] (i.e., the ratio of the Rayleigh backscattering power to the SpBS power), or its inverse (representing the normalized SpBS power), is commonly used instead of SpBS intensity in order to compensate for the fiber loss. The changes due to strain $(\Delta \varepsilon)$ and temperature $(\Delta T)$ impacting the BFS $\left(\Delta \nu_{B}\right)$ and the normalized SpBS power $\left(\Delta P_{B}\right)$ can be described by the following matrix equation [4]:

$$
\left[\begin{array}{l}
\Delta \nu_{B} \\
\Delta P_{B}
\end{array}\right]=\left[\begin{array}{ll}
C_{\nu_{B} \varepsilon} & C_{\nu_{B} T} \\
C_{P_{B} \varepsilon} & C_{P_{B} T}
\end{array}\right]\left[\begin{array}{c}
\Delta \varepsilon \\
\Delta T
\end{array}\right]
$$

where $C_{\nu B \varepsilon}$ and $C_{\nu \mathrm{BT}}$ are the strain and temperature coefficients for BFS, $C_{\mathrm{PB} \varepsilon}$ and $C_{\mathrm{PBT}}$ are the coefficients for $\mathrm{SpBS}$ power $\left(C_{\nu B \varepsilon}=0.046 \mathrm{MHz} / \mu \varepsilon, C_{\nu \mathrm{BT}}=1.07 \mathrm{MHz} /{ }^{\circ} \mathrm{C}\right.$, $C_{\mathrm{PB} \varepsilon}=-8 \times 10^{-4 \%} / \mu \varepsilon, C_{\mathrm{PBT}}=0.36 \% /{ }^{\circ} \mathrm{C}$ [4]). Simultaneous distributed temperature and strain estimation is then possible in principle by inverting (1). To estimate the final temperature and strain sensing accuracy, the respective root-meansquare (rms) errors, $\delta \varepsilon$ and $\delta T$, can be used. These are given by [6]

$$
\begin{array}{r}
\delta \varepsilon=\frac{\left|C_{P_{B} T}\right| \delta \nu_{B}+\left|C_{\nu_{B} T}\right| \delta P_{B}}{\left|C_{\nu_{B} \varepsilon} C_{P_{B} T}-C_{P_{B} \varepsilon} C_{\nu_{B} T}\right|} \\
\delta T=\frac{\left|C_{P_{B} \varepsilon}\right| \delta \nu_{B}+\left|C_{\nu_{B} \varepsilon}\right| \delta P_{B}}{\left|C_{\nu_{B} \varepsilon} C_{P_{B} T}-C_{P_{B} \varepsilon} C_{\nu_{B} T}\right|}
\end{array}
$$

where $\delta \nu_{B}$ and $\delta P_{B}$ are the rms errors in BFS and normalized SpBS power measurements, respectively. In SpBS-based strain and temperature sensing, coherent detection schemes have to be used in order to accurately measure BFS and normalized intensity parameters. In such schemes, the SpBS signal is mixed with an optical local oscillator (OLO) before photodetection, allowing for higher sensitivity and dynamic range compared to simpler direct-detection receiver scheme.

Recently, it has been shown that optical pulse coding, and in particular Simplex coding [7], can be effectively applied to direct-detection Brillouin intensity measurement providing significant performance improvement in temperature sensing due to enhancement in signal-to-noise ratio (SNR), especially at low 


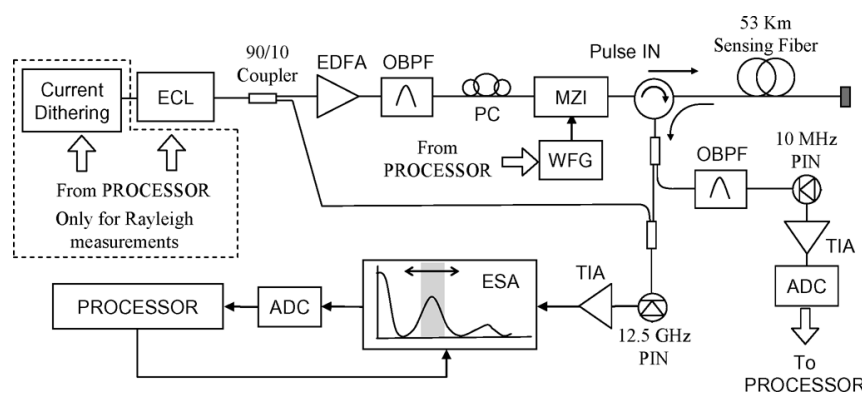

Fig. 1. Experimental setup of implemented distributed Brillouin sensor for simultaneous strain and temperature measurements.

input peak pulse power levels. The SNR improvement at a given distance can be quantified by the coding gain, defined as the ratio of the SNR obtained with coded pulses to the SNR obtained with single pulses, and for the same number of acquired traces. The coding gain in case of Simplex coding can be expressed as $(L+1) /(2 \sqrt{ } L)$ [7], where $L$ is the code length, indicating that most of the coding gain advantage is obtained for moderate code lengths (in our experiments $L=127$ ). Pulse coding can thus lead to more accurate coherent detection of Brillouin power and BFS, and to a notably enhanced accuracy in simultaneous strain and temperature sensors.

\section{EXPERIMENTAL SETUP}

Fig. 1 reports the experimental setup implemented for simultaneous strain and temperature sensing. The light from an external cavity laser (ECL) operating at $1555.0 \mathrm{~nm}$ is split into two parts, the OLO and the probe signal, by using a $10 \% / 90 \%$ optical splitter. The continuous-wave $(\mathrm{CW})$ probe signal is optically amplified by an erbium-doped fiber amplifier (EDFA) and filtered by an optical bandpass filter [(OBPF) 0.5 -nm bandwidth] in order to reduce amplified spontaneous emission (ASE) noise. By using a polarization controller (PC) and a Mach-Zehnder interferometer (MZI), the CW light is then intensity-modulated with either 127-bit Simplex codes or single pulses. The single pulse duration is $320 \mathrm{~ns}$, corresponding to an attainable spatial resolution of $32 \mathrm{~m}$. The optical pulses (10-mW peak power) are then launched into $53 \mathrm{~km}$ of dispersion-shifted fiber (DSF) through an optical circulator which is also used to couple into the receiver the backscattered light from the fiber, including the Brillouin and Rayleigh components. A small fraction of the scattered light is split out for measuring the Rayleigh backscattering power trace; this is accomplished through an OBPF and a PIN photodiode followed by a transimpedance amplifier (TIA) and analog-digital conversion (ADC). Note that, when measuring the Rayleigh component, coherent Rayleigh noise (CRN), due to the narrowband optical source required in Brillouin sensors, is properly reduced down to $0.4 \%$ using both laser current dithering and $\lambda$-averaging over $0.5 \mathrm{~nm}$. The backscattered light is mixed to the OLO, and then coupled to a PIN photodiode followed by a TIA (12.5-GHz bandwidth). Note that the Rayleigh backscattering component does not affect the mixing process in our case because its peak power is in fact well below the OLO power. In order to accurately obtain the spectral shape of the SpBS light, OTDR traces at different spectral components within the Brillouin gain bandwidth are measured by using an

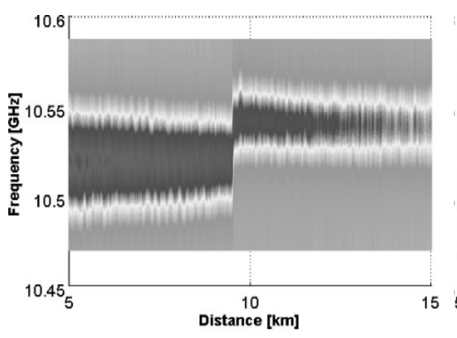

(a)

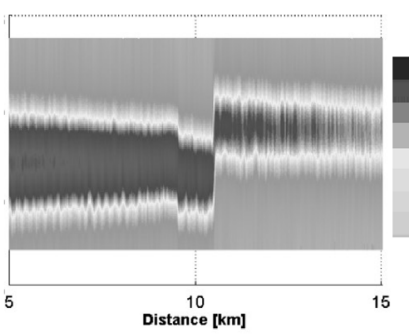

(b)
Fig. 2. Example of measured Brillouin power spectrum shape: (a) whole fiber at $300 \mathrm{~K}$, and (b) with 1-km-long fiber spool at $273 \mathrm{~K}$.

electric spectrum analyzer (ESA) operating in zero-span mode and tuned to different central frequencies (around $11 \mathrm{GHz}$ ). The largest available resolution bandwidth in the ESA ( $3 \mathrm{MHz})$ leads to a minimum spatial resolution of $32 \mathrm{~m}$.

The Brillouin spectral shape along the sensing fiber is then obtained by stepping over 40 neighbouring central frequency values (3-MHz steps, starting from $10.47 \mathrm{GHz}$ ), within the Brillouin gain bandwidth, and measuring the corresponding traces. In order to estimate the BFS, the spectrum at each fiber location is fitted with a Lorentzian curve by using a least-squares algorithm. Normalized Brillouin power is obtained by integrating the Brillouin spectral power, and then using the measured Rayleigh scattering trace. Fig. 2(a) shows a contour plot representing the BFS obtained with this technique at room temperature $(300 \mathrm{~K})$. Fig. 2(b) refers to the case when a spool of 1-km length, at a distance of $9.5 \mathrm{~km}$, is cooled down to $273 \mathrm{~K}$ by using a temperature-controlled chamber. The discontinuity in the BFS shown in Fig. 2(a) is due to slightly different material composition of the different fiber spools, not representing any temperature variation. However, this effect is compensated by using this trace as a reference for temperature estimation. In Fig. 2(b), we can clearly notice a shift of $\sim 30 \mathrm{MHz}$ in the BFS induced by $\sim 27 \mathrm{~K}$ temperature change. Note that similar BFS would also be observed when applying a strain of $\sim 600 \mu \varepsilon$. In order to distinguish between temperature and strain, the normalized Brillouin power should be used as well, following the prescriptions in Section II.

\section{RESULTS}

In this section, we compare the performance of conventional and Simplex-coded DFS employing simultaneous BFS and Brillouin intensity measurements for combined strain and temperature sensing. Note that strain and temperature variations are not necessarily applied in performance analysis, which aims at evaluating the sensor performance in terms of attainable rms noise and resolution parameters, depending ultimately on the SNR behaviour of the measured traces. Fig. 3 shows the accuracy (in terms of rms error) achieved for normalized SpBS power [Fig. 3(a)] and BFS measurements [Fig. 3(b)] versus distance, comparing the case of 127 bit Simplex-coding to the conventional Brillouin sensor case. Note that the SNR benefits of pulse coding are expected to be the same (in terms of $G_{\mathrm{COD}}$ ) also for shorter pulse durations (the lower limit is $\sim 10 \mathrm{~ns}$, below which broadening of Brillouin gain spectrum starts to occur). The experimental SNR enhancement provided by coding in normalized Brillouin power results in $7.4 \mathrm{~dB}$, which is in agreement with the theory $\left(G_{\mathrm{COD}}=7.54 \mathrm{~dB}\right)$ and represents a reduction in rms error, as shown in Fig. 3(a). This SNR improvement also 
(a)

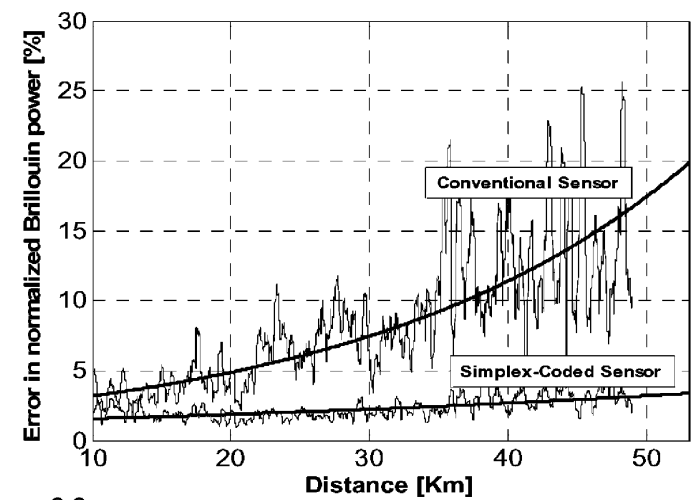

(b)

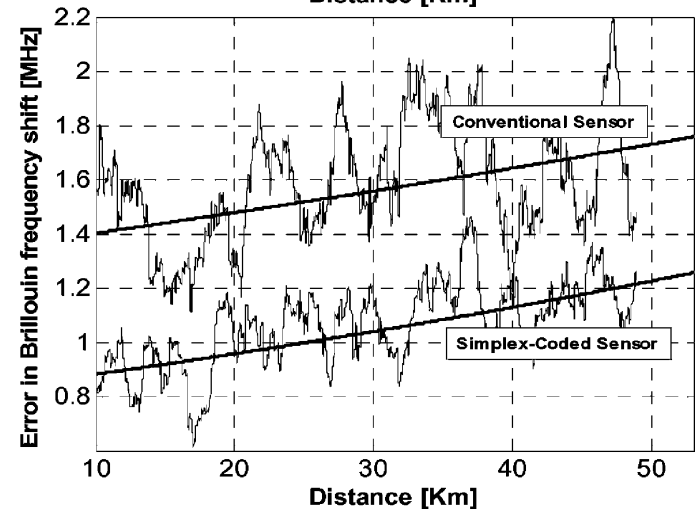

Fig. 3. Measurement rms error in (a) Brillouin normalized power and (b) frequency shift versus distance, for conventional and Simplex-coded Brillouin sensor.

helps in reducing the rms noise in the $\mathrm{BFS}\left(\delta \nu_{B}\right)$, although to a smaller extent, since $\delta \nu_{B}$ depends on the SNR according to

$$
\delta \nu_{B}=\frac{\Delta \nu_{B}}{\sqrt{2}(\mathrm{SNR})^{1 / 4}}
$$

where $\Delta \nu_{B}$ is the Brillouin light linewidth [2]. Fig. 3(b) points out the impact of optical pulse coding on the BFS measurement accuracy; the improved SNR allows for an average accuracy improvement of $\sim 1.5$ times on the BFS compared to conventional Brillouin sensor. Most notably, referring to the same accuracy, Fig. 3(b) also indicates that the use of coding allows for an improvement in the sensing range of $\sim 7.5 \mathrm{~dB}$ compared to single-pulse. Note that the comparisons in Fig. 3(a) and (b) are performed considering the same total averaging number. Thus, to reduce the receiver electrical noise, every trace is averaged $2^{13}$ times in single-pulse case and $2^{13} / 127$ times for each codeword in case of coding.

When calculating the final strain and temperature resolution from (1) and (2), the accuracy levels in both Brillouin power and BFS measurements have an impact. Fig. 4(a) and (b) show a comparison of the achievable temperature and strain resolutions with single-pulse and 127-bit Simplex coding, when sensing both physical variables simultaneously. For the single-pulse case, a poor temperature resolution $(52 \mathrm{~K})$ is achieved near the fiber-end $(53 \mathrm{~km})$, mainly due to the low peak power used in the experiment $(10 \mathrm{~mW})$. Such a resolution is improved down to $8.8 \mathrm{~K}$ when using 127-bit Simplex coding, mainly thanks to the SNR improvement in Brillouin power measurements. Strain resolution is also significantly improved by the use of coding, as shown in Fig. 4(b) (e.g., indicating an enhancement (a)

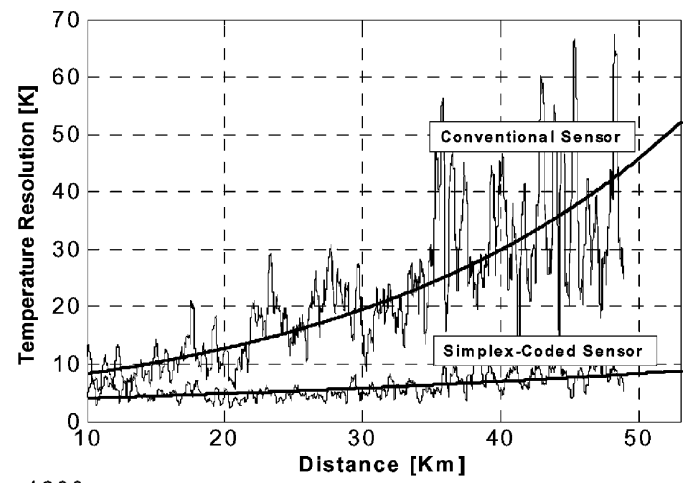

(b)

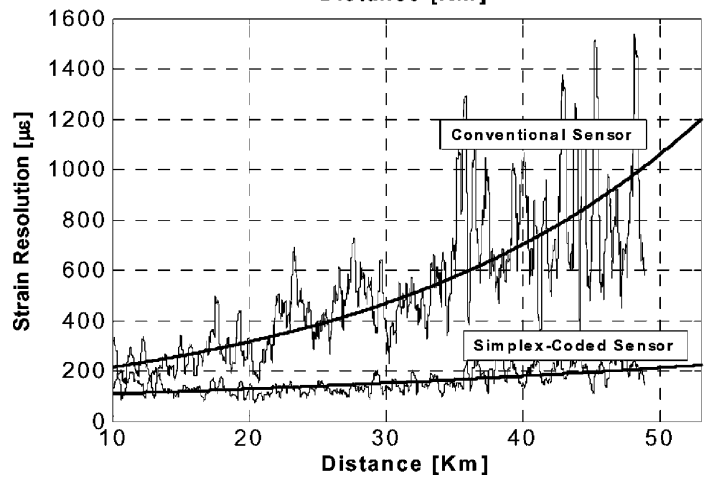

Fig. 4. (a) Temperature resolution and (b) strain resolution versus distance, for conventional and Simplex-coded Brillouin-based sensor.

of strain resolution from $\sim 1200 \mu \varepsilon$ down to $\sim 220 \mu \varepsilon$ at $53 \mathrm{~km}$ ). Note that the most notable benefits of coding in simultaneous strain and temperature Brillouin-based sensors appear when comparing the range enhancement achieved for the same resolution values. Pulse coding actually allows for a significant increase in resolution, for both strain and temperature, which from Fig. 4 appears equal to nearly $40 \mathrm{~km}$ in the sensing range, or equivalently an increase of $\sim 7.5 \mathrm{~dB}$ one-way (and $\sim 15 \mathrm{~dB}$ two-way) in the span budget.

In conclusion, we have shown that pulse coding, applied to SpBS measurement, allows for accurate simultaneous strain and temperature sensing, providing a high-performance and costeffective solution, avoiding the use of optical pulse amplification and high peak power values.

\section{REFERENCES}

[1] Nature Photonics, "Optical-fibre sensors," Tech. Focus, vol. 2, pp. 143-158, 2008.

[2] T. Horiguchi, K. Shimizu, T. Kurashima, M. Tateda, and Y. Koyamada, "Development of a distributed sensing technique using Brillouin scattering," J. Lightw. Technol., vol. 13, no. 7, pp. 1296-1301, Jul. 1995.

[3] P. C. Wait and T. P. Newson, "Landau Placzek ratio applied to distributed fiber sensing," Opt. Commun., vol. 122, pp. 141-146, 1996.

[4] S. M. Maughan et al., "Simultaneous distributed fibre temperature and strain sensor using microwave coherent detection of spontaneous Brillouin backscatter," Meas. Sci. Technol., vol. 12, pp. 834-842, 2001.

[5] A. Minardo et al., "A reconstruction technique for long-range stimulated Brillouin scattering distributed fibre-optic sensors: Experimental results," Meas. Sci. Technol., vol. 16, pp. 900-908, 2005.

[6] J. D. C. Jones, "Review of fibre sensor techniques for temperaturestrain discrimination," in Proc. Optical Fiber Sensors Conf., Washington, DC, 1997, vol. 16, OSA Tech. Dig. Series, pp. 36-39, Paper OFS-12.

[7] M. A. Soto, P. K. Sahu, G. Bolognini, and F. Di Pasquale, "Brillouinbased distributed temperature sensor employing pulse coding," IEEE Sensors J., vol. 8, no. 3, pp. 225-226, Mar. 2008. 